\title{
Evaluation of correlation between ocular manifestations with CD4+ count in HIV patients: a cross-sectional study
}

\author{
Rekha K.R.
}

Department of Ophthalmology, Vydehi Medical College and Research Center, Bangalore, India

\begin{abstract}
Introduction: With the introduction of highly active antiretroviral therapy (HAART), improved quality of life and reduced mortality in human immunodeficiency virus (HIV)-infected population have increased. The importance of visual acuity, in any individual is obvious, and heightened importance in those afflicted by disorders is even more crucial. In this context, this study reports details of a comprehensive ocular evaluation in a HIV population, and its' correlation with severity of the disease. The aim of the study was to determine the incidence of ocular manifestation and correlate CD4+ count levels, with incidence of ocular manifestations in HIV-positive patients.

Material and methods: This was a cross-sectional study that evaluated ophthalmic manifestations in patients diagnosed with HIV infection in an urban ophthalmological center. Detailed ophthalmological examination was conducted. Various parameters of the disease and severity of HIV were estimated and correlated with ophthalmological findings.

Results: In 2 years, 300 HIV-positive (229 males) patients, mostly aged between 30 and 40 years, were assessed. $69(23 \%)$ patients were found to have ocular manifestation, $73.9 \%$ were in the range of 0-5 years of HIV duration, 28 (9.3\%) patients presented with anterior segment and $62(26.6 \%)$ with posterior segment pathology, like HIV retinopathy. Cytomegalovirus retinitis poor visual acuity was observed in females (20.5\%), aged 0-35 years, with HIV duration of 0-5 years and CD4+ count less than 200 .
\end{abstract}

Conclusions: The focus on a comprehensive ocular evaluation, irrespective of visual acuity and possibility of improvement in ocular follow-up of HIV-infected patients needs to be emphasized.

HIV AIDS Rev 2021; 20, 4: 264-269

DOI: https://doi.org/10.5114/hivar.2021.111874

Key words: CD4+ counts, HIV, cytomegalovirus (CMV) retinitis, HIV retinopathy, HAART.

\section{Introduction}

Acquired immunodeficiency syndrome (AIDS) is a fatal multisystem syndrome characterized by profound disruption of immune system, showing tendency for various opportunistic infections and neoplasms in patients with a CD4+ T-lymphocyte count rate of less than 200 cells $/ \mathrm{ml}$.

Address for correspondence: Dr. Rekha K.R.,

Department of Ophthalmology, Vydehi Medical College

and Research Center, Door number 96, 2nd cross, 5th block,

Banashankari, Bangalore 85, India,

e-mail: rekhakrathnaiah@gmail.com

AIDS is caused by either of two human immunodeficiency viruses, i.e., HIV-1 or HIV-2 [4]. AIDS was firstly recognized in 1981 as outbreaks of usual opportunistic infections in homosexual men in three American cities. The disease is now a global pandemic, with a total of 75.7 million people infected with human immunodeficiency virus (HIV) since its' start. The UNAIDS estimated that about 38 million people

\section{Article history:}

Received: 30.12 .2020

Received in revised form: 19.03.2021

Accepted: 21.03.2021

Available online: 30.11 .2021
International Journal of HIV-Related Problems

HIV \& AIDS

R e vi e w 
globally were living with HIV in 2019, with 36.2 million adults. Presently, the incidence of new HIV infections have reduced by $40 \%$ since the peak in 1996 [1]. 1.7 million people became newly HIV-infected, and about 25.4 million people were on antiretroviral therapy in 2019 in India, constituting third largest HIV population in the world.

In 2017, HIV prevalence in India among adults (aged, 15-49) was estimated at $0.2 \%$, and this equates to 2.1 million people living with HIV overall. A small decrease of India's HIV epidemic was noted between 2010 and 2017. New HIV infections declined by $27 \%$, with $56 \%$ of AIDSrelated deaths. HIV prevalence was higher among men $(0.25 \%)$, with $0.19 \%$ prevalence in women living with HIV, resulting in 88,000 of new HIV infections and 69,000 of AIDS-related deaths [2]. Karnataka has 1.75 lakh people with HIV under active care, according to data of Karnataka State AIDS Prevention Society. Ocular manifestation may be the primary presentation of HIV. The eye is an organ with wide spectrum of HIV-related manifestation, and the disease can present with adnexal, anterior segment, posterior segment, orbital, and neuro-ophthalmic manifestation [5].

Ocular manifestation is present in up to $73 \%$ of AIDS patients. Most common include HIV retinopathy, cytomegalovirus (CMV) retinitis, conjunctival Kaposi sarcoma, herpes zoster ophthalmicus retinal toxoplasmosis, choroidal Pneumocystis jiroveci infection, herpes simplex and herpes zoster retinitis (acute retinal necrosis, ARN), and cryptococcal choroiditis. Iritis occur in association with CMV oral rifabutin, intravenous cidofovir, choroidal infection with Cryptococcus, Pneumocystis, Mycobacterium tuberculosis, Aspergillus, and Toxoplasma. HIV retinopathy manifestation includes cotton wool spots, intraretinal hemorrhage micro-aneurysm, and exudates. Cotton-wool spots are the most common ocular lesion occurring in $25-50 \%$ of AIDS patients, and are confined to the posterior pole near the optic disc. Retinal hemorrhages are seen in up to $30 \%$ of AIDS patients in forms of flame-shaped lesions in the posterior pole, dot-blot hemorrhages, punctate intraretinal hemorrhages peripherally, and Roth's spots. CMV retinitis is the most common ocular opportunistic infection occurring in approximately $20-40 \%$ of HIV-infected patients, considered as the leading cause of $90 \%$ of blindness associated with AIDS worldwide. CMV and HIV are found to transactivate each other, leading to a greater degree of immune suppression found in HIV patients, a possible explanation for CMV retinitis appearing more commonly in HIV patients as opposed to non-HIV cases. Additional risk factors include higher CD8+ T-lymphocytes counts, lack of antiretroviral therapy, and male gender. It has been observed that HIV patients on highly active antiretroviral therapy (HAART) with lower than 10-fold decrease in HIV viral loads during the treatment period, are at elevated risk for opportunistic infection, including reactivation of CMV retinitis. HIV-related retinopathy is an ocular risk factor for CMV retinitis development [7]. The advent of HAART have declined the incidence and severity of pattern of HIV-related ocular manifestations, although the prevalence remains high, partly due to increasing survival rates in AIDS patients. In HAART, ocular manifestation present in different patterns and newer manifestation, including dry eye syndrome, vernal keratoconjunctivitis, and ocular surface squamous necrosis seen in significant proportion. CMV retinitis is still the most common ocular opportunistic infection, followed by toxoplasmosis and syphilis. CMV retinitis can be prevented with HAART optimization in early course of HIV disease. Despite current therapeutic regimens and the increase of life expectancy of HIV patients, the progression of retinitis often occurs, and as survival increases, ocular complications may occur even more frequently overtime. Immune recovery uveitis is an increase in intra-ocular inflammation following HAART and occurs in immune-deficient patients with prior cytomegalovirus retinitis. This happen due to recovery of immune response, specific to residual cytomegalovirus antigens in the eye. MDR-TB is a new scope in immune recovery uveitis, causing vision threatening ocular manifestation [7]. In HAART era, the role of an ophthalmologist is pivotal. It does not end with just diagnosing and managing patients, but to minimize and prevent long-term complications of irreversible visual loss, thus improving quality of life of these patients [6]. Therefore, an understanding of the constantly changing pattern of ocular manifestation of HIV infection due to increased survival of HIV-infected patients as well as management of HIV patients on HAART, can help in early diagnosis and supervision of ocular and general morbidity of AIDS. The present study emphasized the need of developing comprehensive ocular evaluation, and correlation of CD4+ count level and ocular manifestation of HIV infection in HAART era.

The objectives of the study were to determine the incidence of ocular manifestation in HIV-positive patients, and to correlate the duration of HIV disease and CD4+ count levels with the incidence of ocular manifestations.

\section{Material and methods}

This was a cross-sectional study that evaluated ophthalmic manifestations in a group of patients with diagnosed HIV infection in an urban ophthalmological center. HIV testing and $\mathrm{CD} 4+$ count level estimation were done by a physician, using standard methods according to national guidelines. Moreover, a thorough physician evaluation was performed.

Detailed ophthalmological examination included visual acuity estimation using Snellen chart and refraction. Thorough anterior segment examination was performed using a slit lamp, and detailed fundus examination was done using indirect ophthalmoscope (+90D or $+78 \mathrm{D})$. The examining consultant determined the presence or absence of specific condition based on visualized clinical findings. Details of demographic characteristics of the patient, HIV status (duration of disease, CD4+ count, receipt of HAART) were recorded. Patients with severe debilitating disorders and coexistence of other systemic disorders, such as hypertension or diabetes, which are likely to be associated with ophthalmologic manifestations rather than HIV, were excluded. 
The collected data were analyzed using IBM. SPSS statistics software, 23.0 version. To describe data descriptive statistics frequency analysis, percentage analysis were applied for categorical variables, with mean and standard deviation (SD) used as continuous variables. To find significance in categorical data, $\chi^{2}$ test was used. In the above statistical tool, probability value of 0.05 was considered as statistically significant.

\section{Results}

In Table 1, demographic characteristics of the $300 \mathrm{HIV}$ positive patients evaluated in the present study are presented. 229 patients were males, aged between 30 and 40 years, and with duration of the disease of less than 5 years (Table 1). All the patients reported a heterosexual mode of transmission. $61(20.34 \%)$ patients in our study had CD4+ count less than 200 cells $/ \mathrm{mm}^{3}$. In Table 2, prevalence of ocular manifestations based on gender and age distribution of 300 patients are shown. $69(23 \%)$ patients were found to present with an ocular manifestation. Of these, 28 (9.3\%) cases were found to have an anterior segment and ocular adnexal lesion, and $62(26.6 \%)$ were observed to have a posterior segment pathology. Ocular manifestations were more common in males (60 patients, $20 \%$ ), and prevalence in males

Table 1. Demographic characteristics of present study

\begin{tabular}{|c|c|}
\hline Factor & $n(\%)$ \\
\hline \multicolumn{2}{|l|}{ Gender } \\
\hline Male & $229(76.34)$ \\
\hline Female & $71(23.66)$ \\
\hline \multicolumn{2}{|l|}{ Age (years) } \\
\hline$<30$ & $88(29.34)$ \\
\hline $30-40$ & $154(51.34)$ \\
\hline $40-50$ & $39(13.00)$ \\
\hline$>50$ & $19(6.34)$ \\
\hline \multicolumn{2}{|c|}{ Duration of HIV (years) } \\
\hline $0-5$ & $270(90.00)$ \\
\hline $6-10$ & $30(10.00)$ \\
\hline \multicolumn{2}{|c|}{ CD4 count (cells/mm³) } \\
\hline$\leq 200$ & $61(20.34)$ \\
\hline $201-500$ & $36(12.00)$ \\
\hline $501-1000$ & $44(14.66)$ \\
\hline$>1000$ & $159(53.00)$ \\
\hline
\end{tabular}

was higher than prevalence in females (9 patients, 3\%), with $p=0.0329$ (Table 2). Ocular manifestation was more common in age group of more than 35 years (33\% vs. 18\%) $(p=0.125$, Table 2).

Table 3 describes prevalence of vision based on gender, age, duration of HIV infection, and CD4+ count, with females $(20.5 \%)$ having a higher proportion of poor visual acuity than males (2.1\%). Those with age below 35 years had a higher proportion of poor visual acuity (9.5\%) compared to older than 35 years $(0 \%)$ patients. Those with poor visual acuity tended to present ocular manifestations in the first

Table 2. Prevalence of ocular manifestations based on gender and age distribution

\begin{tabular}{|c|c|c|c|}
\hline Factor & $\begin{array}{c}\text { Ocular } \\
\text { manifestation } \\
n(\%)\end{array}$ & $\begin{array}{c}\text { No ocular } \\
\text { manifestation } \\
n(\%)\end{array}$ & $p$-value \\
\hline \multicolumn{4}{|l|}{ Gender } \\
\hline Male & $60(20)$ & $172(57.3)$ & \multirow[t]{2}{*}{0.0329} \\
\hline Female & $9(3)$ & 59 (19.6) & \\
\hline \multicolumn{4}{|l|}{ Age (years) } \\
\hline $0-35$ & $36(18)$ & $164(82)$ & \multirow[t]{2}{*}{0.0125} \\
\hline$>36$ & $33(33)$ & $67(67)$ & \\
\hline
\end{tabular}

Table 3. Prevalence of vision based on gender age duration of HIV infection and CD4 count

\begin{tabular}{|c|c|c|c|}
\hline Vision & $\begin{array}{c}\text { Normal } \\
6 / 6-6 / 60\end{array}$ & $\begin{array}{l}\text { Abnormal less } \\
\text { than } 6 / 60---P L-\end{array}$ & $p$-value \\
\hline \multicolumn{4}{|l|}{ Gender } \\
\hline Male & 227 & $5(2.1)$ & \multirow[t]{2}{*}{0.0001} \\
\hline Female & 54 & $14(20.5)$ & \\
\hline \multicolumn{4}{|l|}{ Age (years) } \\
\hline$<35$ & 181 & 19 & \multirow[t]{2}{*}{0.0006} \\
\hline$\geq 36$ & 100 & 0 & \\
\hline \multicolumn{4}{|c|}{ Duration (years) } \\
\hline $0-5$ & 223 & $19(7.8)$ & \multirow[t]{2}{*}{0.0305} \\
\hline $6-10$ & 58 & 0 & \\
\hline \multicolumn{4}{|c|}{ CD4 count (cells/mm³) } \\
\hline$<200$ & 48 & $13(21.3)$ & \multirow[t]{2}{*}{0.0001} \\
\hline$\geq 200$ & 233 & $6(2.5)$ & \\
\hline
\end{tabular}

Table 4. Distributions of ocular lesions based on duration of disease

\begin{tabular}{l|c|c|c}
\hline Duration & $0-5$ years & $6-10$ years & $\chi^{2}$ test $p$-value statistically significant \\
\hline Ocular manifestion & 51 & 18 & 0.0001 \\
\hline Infectious etiology & 46 & 17 & 0.0001 \\
\hline Posterior segment ocular manifestation & 47 & 15 & 0.0001 \\
\hline
\end{tabular}


Table 5. Distributions of ocular lesions based on CD4 count (cells $/ \mathrm{mm}^{3}$ )

\begin{tabular}{l|c|c|c}
\hline CD4 count & Ocular manifestation & Infectious etiology & Posterior segment ocular manifestation \\
\hline$<200$ & 43 & 42 & 40 \\
\hline $200-500$ & 14 & 12 & 14 \\
\hline $500-1000$ & 3 & 1 & 2 \\
\hline$>1000$ & 9 & 8 & 6 \\
\hline
\end{tabular}

Table 6. Distribution of individual ocular manifestations and their correlation with CD4 count (cells/mm³) with $p$-value $(<0.005$ is significant)

\begin{tabular}{l|c|c|c|c|c}
\hline Diagnosis & $<200$ & $201-500$ & $501-1000$ & $>1000$ & $p$-value \\
\hline Herpes zoster ophthalmicus & 1 & 0 & 0 & 1 & 0.877 \\
\hline Conjunctival vasculopathy & 3 & 0 & 0 & 3 & 0.433 \\
\hline Anterior uveitis & 15 & 4 & 0 & 0 & 0.005 \\
\hline TB conglomerate tubercle & 1 & 0 & 0 & 0 & 0.092 \\
\hline HIV retinopathy & 20 & 7 & 1 & 4 & 0.005 \\
\hline CMV retinitis & 20 & 2 & 0 & 0 & 0.005 \\
\hline Toxoretinochoroidits & 1 & 1 & 0 & 0 & 0.105 \\
\hline ARN/PORN & 1 & 1 & 0 & 0 & 0.092 \\
\hline Tb choroditis & 4 & 2 & 1 & 1 & 0.010 \\
\hline Posterior uveitis & 14 & 3 & 0 & 0 & 0.005 \\
\hline Vitreous hemorrhage & 0 & 0 & 3 & 0 & 0.006 \\
\hline
\end{tabular}

few years rather than in later. Patients with CD4+ count less than 200 cells $/ \mathrm{mm}^{3}$ had a much poor visual acuity compared to patients with $\mathrm{CD} 4+$ count higher than 200 cells $/ \mathrm{mm}^{3}$. Table 4 present distributions of ocular lesions based on duration of disease, in which the relationship between duration of disease and its' influence on visual findings was analyzed. Each of the items (any ocular manifestation, infectious etiology, posterior segment ocular manifestation) was seen more commonly in patients with shorter duration of the disease (Table 4). $73.9 \%$ of the patients had a $0-5$ years of HIV disease duration with a developed ocular manifestation.

Table 5 demonstrates distributions of ocular lesions based on CD4+ count. As shown in Table 5, each of the items (any ocular manifestation, infectious etiology, posterior segment ocular manifestation) were higher in proportion in patients with CD4 count $<200$.

Table 6 shows distribution of individual ocular manifestations and their correlation with $\mathrm{CD} 4+$ count, with a $p$-value $<0.005$ as statistically significant. As shown in Table 6, with a reduction in CD4+ count, HIV retinopathy and CMV retinitis were more common $(p<0.05)$. Ten patients with good visual acuity presented with CMV retinitis, and 3 patients with CD4+ count of 14, 20, and 300 had both HIV retinopathy and CMV retinitis. CD4+ count did not influence the prevalence of Herpes zoster ophthalmicus, conjunctival vasculopathy, TB conglomerate tubercle, toxo-retinochoroiditis, and acute retinal necrosis.

\section{Discussion}

In this cross sectional study, $300 \mathrm{HIV}$-positive patients were evaluated for ocular manifestations. All the patients were on HAART, and CD4+ count estimation was available for all. Ophthalmological examination was performed regardless of their ocular symptoms.

\section{Age}

In our study, $51.34 \%$ the patients were in the age group of 30-40 years, which was similar to studies by Hothi et al., with mean age of 38.6 years, and Shah et al., with mean age of 38.96 years. This was also comparable to Indian national estimates, where the proportion of patients between 20 and 40 years ranges between $46 \%$ and $75 \%$.

\section{Gender}

In our study, males constituted for nearly $76 \%$ and females for $24 \%$ of the participants, which was comparable to a study by Sudarshan et al. (77\% males); however, it was slightly higher than that reported by Shah et al. (68\% males) and Hothi et al. (60\% males). Demographic patterns in the present study were similar to Indian national HIV statistics, and therefore, this study population can be taken as representative. 


\section{Duration of HIV}

In a study by Sudarshan et al., time duration of onset of ocular lesion ranged from 10 days to 10.24 years. This study evaluated correlation of duration of HIV disease and levels of the incidence of ocular manifestations. In our study, a majority (73.9\%) of the patients developed ocular manifestations in the first 5 years of the disease. Also, it was observed that longer duration of HIV did not appear to be a risk factor in ocular lesion causation (statistically significant difference shown in Table 4).

\section{Visual impairment}

Shah et al. reported $6 \%$ of visual impairment due to HIVrelated ocular diseases, including blindness (1\%). Hothi et al. in their study found that $70 \%$ of patients had good vision (> 6/18), 14\% BCVA cases were $<6 / 60$ to CF, and $4 \%$ had defective projection of rays. No patient had visual impairment attributable to HIV retinopathy. In our study, 93.67\% of patients presented with good vision $(>6 / 18)$, whereas $6.3 \%$ had BCVA of 6/60 and PL. In 19 patients, vision was less than hand movements due to retinal detachment, CMV retinitis, and acute retinal necrosis. As mentioned, 10 of 22 patients had good visual acuity and presented with CMV retinitis in peripheral retina, highlighting the fact that all patients with HIV, irrelevant of their visual acuity, should undergo detailed ophthalmological evaluations.

\section{Total prevalence}

In our study, the total prevalence of ocular manifestation was $23 \%$, of which, $9.3 \%$ had anterior segment involvement and $26.6 \%$ had posterior segment ocular manifestation. Similarly, Hothi et al. reported a corresponding proportion of $25.3 \%$, with $20 \%$ adnexal involvement, $28 \%$ anterior segment involvement, and $33 \%$ posterior segment involvement.

\section{Types of lesion: anterior and posterior}

Hothi et al. study showed HIV retinopathy as the most common ocular finding in posterior segment (15.3\%), followed by retinal detachment (12.8\%), CMV retinitis (5.12\%), and tuberculous choroiditis (1\%). Lower prevalence of CMV retinitis was probably due to antiretroviral therapy or due to late presentation as complication, like retinal detachment. Jab et al. observed CMV retinitis as the most frequent ocular finding $(22.7 \%)$. In our study, CMV retinitis and HIV retinopathy were common entities also.

\section{Correlation with CD4}

Out of 69 patients who had any ocular manifestations, 32 were HIV retinopathy and 22 were CMV retinitis. Three patients presented with coexisting disease and their CD4+ counts were 14,20 , and 300 , respectively. The proportion of patients with CD4+ count less than $200 \mathrm{cell} / \mathrm{mm}^{3}$ who presented with ocular manifestation was $51 \%$ in the study by Hothi et al. and $62.3 \%$ in the present study. Patients with a low CD4+ count had a higher occurrence of CMV retinitis, acute retinal necrosis, HIV retinopathy, and tubercular choroiditis (statistically significance shown in Table 5). Thirtytwo cases of HIV retinopathy had CD4+ counts $<100$ cells/ $\mu \mathrm{l}$, and $16(72.7 \%)$ of 22 cases of active CMV retinitis had CD4 counts $<100$ cells/ $\mu$ l. There were no immune recovery uveitis cases in our study, since most of the patients with CMV retinitis were having CD4+ count $<100$ cells $/ \mu$, and active lesions of CMV retinitis were present in posterior pole. Munro et al. observed that HIV retinopathy was associated with lower CD4+ count cell and higher plasma HIV-RNA levels, but was not associated with a non-infectious micro-angiopathy. CWS represent an area of retinal micro-infarction due to compromised circulation, and may allow CMV to enter retina and proliferate. Due to this association with HIV retinopathy and subsequent CMV retinitis, routine monitoring of HIV retinopathy may allow for early diagnosis of CMV retinitis. In our study, the occurrence of toxoplasmosis and tubercular infection was quite less. This was surprising since these diseases are endemic in our country. Epidemiological studies have shown that AIDS patients have very high susceptibility to develop tuberculosis when exposed (16-25 times higher in AIDS patients) [13].

\section{Conclusions}

The present sudsy showed that there is a large proportion of patients who may not present with visual impairment or ophthalmic symptoms, but present with potentially dangerous conditions, including CMV retinitis or other opportunistic infections, especially with low CD4 + T cell count. Therefore, the focus should be on a comprehensive ocular evaluation, irrespective of the level of visual acuity. There is a need for improvement in the guidelines for eye care, screening, and follow-up of HIV-infected patients.

\section{Conflict of interest}

The authors declare no conflict of interest.

\section{References}

1. Global HIV and AIDS statistics - 2020 fact sheet UNAIDS. Available from: https://www.unaids.org/en/resources/fact-sheet\#: :text=25.4\% 20million\%20\%5B24.5\%20million\%E2\%80\%9325.6,infected\%20 with\%20HIV\%20in\%202019 (Accessed: 24.11.2020).

2. HIV and AIDS in India. Available from: https://www.avert. org/professionals/hiv-around-world/asia-pacific/india (Accessed: 24.11.2020)

3. District HIV/AIDS Epidemiological Profiles developed through Data Triangulation. Fact Sheets. Karnataka State. Available from: http://www.naco.gov.in/sites/default/files/Karnatak_DEP.pdf (Accessed: 24.11.2020).

4. Retinal manifestations of the Acquired immunodeficiency syndrome: Diagnosis and Treatment. In: Albert DM, Miller JW, Azar DT, Young LH (eds.). Albert and Jackobiec's Principles and Practice of Ophthalmology. $3^{\text {rd }}$ ed. Elsevier, p. 2095. 
5. Joshi KA, Patel N, Kalita K, et al. Evaluation of fundoscopic findings in HIV positive patients in relation to HARRT and CD4 count. Delhi J Ophthalmol 2018; 29: 34-38.

6. Sudharshan S, Nair N, Curi A, Banker A, Kempen JH. Human immunodeficiency virus and intraocular inflammation in the era of highly active anti retroviral therapy - an update. Indian J Ophthalmol 2020; 68: 1787-1798.

7. Munro M, Yadavalli T, Fonteh C, Arfeen S, Lobo-Chan AM. Cytomegalovirus retinitis in HIV and non-HIV individuals. Microorganisms 2019; 8: 55.

8. Hothi HS, Gohil NR, Parekh NV, Patel SS. A prevalence study of ocular manifestations in HIV positive patients on highly active anti-retroviral therapy. Int J Community Med Public Health 2019; 6: 2950-2954.

9. Sudharshan S, Kaleemunnisha S, Banu AA, et al. Ocular lesions in 1,000 consecutive HIV-positive patients in India: a long-term study. J Ophthalmic Inflamm Infect 2013; 3: 2.

10. Bekele S, Gelaw Y, Tessema F. Ocular manifestation of HIV/AIDS and correlation with CD4+ cells count among adult HIV/AIDS patients in Jimma town, Ethiopia: a cross sectional study. BMC Ophthalmol 2013; 13: 20.

11. Shah SU, Kerkar SP, Pazare AR. Evaluation of ocular manifestations and blindness in HIV/AIDS patients on HAART in a tertiary care hospital in western India. Br J Ophthalmology 2009; 93: 88-90.

12. Jabs DA, Van Natta ML, Holbrook JT, Kempen JH, Meinert CL, Davis MD; Studies of the Ocular Complications of AIDS Research Group. Longitudinal study of the ocular complications of AIDS: 2. Ocular examination results at enrollment. Ophthalmology 2007; 114: 787-793.

13. who.int2020. Tuberculosis and HIV [online]. Available at: https:// www.who.inthiv/topics/tb/about_tb/en/ [Accessed: 21.12.2020]. 